\title{
Uso de aplicativos de tecnologia móvel na rotina de estudantes concluintes de medicina
}

\author{
Use of mobile technology applications in the routine of students during \\ the last year of medicine undergraduate degree
}

\section{Uso de aplicaciones de tecnología movible en la rutina de estudiantes durante el último año de la graduación del grado en medicina}

\author{
Lorena Juliana Marinho Estanislau ${ }^{1, a}$ \\ estanislaulorena@gmail.com | https://orcid.org/0000-0002-8457-2203 \\ Aline Pereira Morais ${ }^{1, a}$ \\ moraisaaline@gmail.com | https://orcid.org/o000-0003-1415-4505 \\ Rodolpho Omena Cabral ${ }^{1, a}$ \\ rodolphoomena@gmail.com | https://orcid.org/0000-0002-8317-4123 \\ José Eudes de Lorena Sobrinho ${ }^{2, b}$ \\ eudeslorena@hotmail.com | https://orcid.org/0000-0001-7820-735X \\ Suélem Barros de Lorena ${ }^{1, b}$ \\ suelem.barros@fps.edu.br | https://orcid.org/000o-0001-8664-9967 \\ ${ }^{1}$ Faculdade Pernambucana de Saúde. Recife, PE, Brasil. \\ ${ }^{2}$ Universidade de Pernambuco, Faculdade de Ciências Médicas. Recife, PE, Brasil. \\ a Graduando em Medicina pela Faculdade Pernambucana de Saúde. \\ b Doutorado em Saúde Pública pela Fundação Oswaldo Cruz.
}

\section{Resumo}

O uso de aplicativos vem crescendo na área da saúde, tornando-se rotina diária na prática dos profissionais e estudantes. Portanto, conhecer benefícios e riscos de seu uso faz-se necessário. O objetivo do estudo aqui apresentado é verificar opiniões e atitudes relatadas por estudantes de medicina a respeito do uso de aplicativos no cotidiano ambulatorial de um hospital-escola. Trata-se de um estudo transversal desenvolvido, em 2017, em Recife, Pernambuco, Brasil, no qual se utilizaram questionários autoaplicáveis presencialmente em uma população de 73 estudantes concluintes do curso de medicina. Os resultados revelaram que a maioria (95,9\%) dos estudantes referiram possuir smartphone e 98,6\% afirmaram utilizar dispositivos móveis com intuito acadêmico. A finalidade mais comum do uso de aplicativos foi a de consulta de fármacos existentes $(93,2 \%)$ e quase a totalidade dos estudantes $(98,6 \%)$ confia nos aplicativos utilizados. Porém, foi questionado se o uso na rotina interfere na relação profissional-paciente. Concluímos que o uso de aplicativos é uma realidade crescente, mas que ainda há discordâncias sobre seus benefícios e malefícios.

Palavras-chave: Aplicativos móveis; Estudantes de medicina; Smartphone; Sistemas de apoio a decisões clínicas; Educação médica. 


\begin{abstract}
The use of applications has been growing in the field of health, becoming a daily routine in the practice of professionals and students. Therefore, knowing the benefits and risks of its use is necessary. The study presented here aims to verify the opinions and attitudes reported by medical students about the use of applications in the outpatient routine in a teaching hospital. It is a cross-sectional study and it was developed in 2017 in Recife, in the state of Pernambuco, Brazil, using a self-administered questionnaire asked by 73 students of the last year of medicine undergraduate degree. The results reveal that the majority (95.9\%) of the students reported having a smartphone and $98.6 \%$ specified that they use mobile devices for academic purposes. The most common purpose of using applications was to consult existing drugs (93.2\%) and almost all students (98.6\%) believe that the applications used are reliables. However, there are doubts if routine use interferes with the professional-patient relationship. We concluded that the use of applications is growing, but there are still disagreements about its benefits and harms.
\end{abstract}

Keywords: Mobile applications; Medical students; Smartphone; Support to clinical decision; Medical education.

\title{
Resumen
}

El uso de aplicaciones viene creciendo en el área de la salud y se ha convertido en rutina diaria en la práctica de los profesionales y estudiantes. Por lo tanto, conocer los beneficios y riesgos de su uso se hace necesario. El objetivo del estudio presentado aquí es verificar opiniones y actitudes relatadas por estudiantes de medicina acerca del uso de aplicaciones en el cotidiano del ambulatorio de un hospital escuela. Un estudio transversal ha sido desarrollado en 2017 en Recife, en el estado de Pernambuco, Brasil, utilizando un cuestionario autoaplicable presencialmente en 73 estudiantes del último año de la graduación del grado en medicina. Los resultados mostran que la mayoría (95,9\%) de los estudiantes mencionó poseer teléfono inteligente y 98,6\% afirmaron utilizar dispositivos movibles con intención académica. La finalidad más común del uso de aplicaciones fue la de consulta de fármacos existentes $(93,2 \%)$ y casi la totalidad de los estudiantes (98,6\%) confía en las aplicaciones utilizadas. Sin embargo, ha sido cuestionado si el uso en la rutina interfiere en la relación profesional-paciente. Nosotros concluimos que el uso de aplicaciones es creciente, pero todavía hay desacuerdos sobre sus beneficios y maleficios.

Palabras clave: Aplicaciones movibles; Estudiantes de medicina; Teléfono inteligente; Sistemas de apoyo a decisiones clínicas; Educación médica.

\begin{abstract}
Contribuição dos autores:
Concepção e desenho do estudo: Suélem Barros de Lorena, Lorena Juliana Marinho Estanislau.

Aquisição, análise ou interpretação dos dados: Aline Pereira Morais, Lorena Juliana Marinho Estanislau, Rodolpho Omena Cabral.

Redação do manuscrito: Aline Pereira Morais, Lorena Juliana Marinho Estanislau, Rodolpho Omena Cabral.

Revisão crítica do conteúdo intelectual: José Eudes de Lorena Sobrinho, Suélem Barros de Lorena.
\end{abstract}

Declaração de conflito de interesses: não houve.

Fontes de financiamento: não houve.

Considerações éticas: o artigo foi aprovado pelo Comitê de Ética da Faculdade Pernambucana de Saúde. CAAE: 56802116.8.0000.5569. Número do parecer: 1.616.223.

Agradecimentos/Contribuições adicionais: não há.

Histórico do artigo: submetido: 30 out. 2018 | aceito: 16 maio 2019 | publicado: 12 set. 2019.

Apresentação anterior: não houve.

Licença CC BY-NC atribuição não comercial. Com essa licença é permitido acessar, baixar (download), copiar, imprimir, compartilhar, reutilizar e distribuir os artigos, desde que para uso não comercial e com a citação da fonte, conferindo os devidos créditos de autoria e menção à Reciis. Nesses casos, nenhuma permissão é necessária por parte dos autores ou dos editores. 


\section{Introdução}

Com as transformações iniciadas pela Revolução Industrial, a era da informatização vem se expandindo de modo bastante acelerado e, ao longo da última década, nota-se um grande crescimento no mercado de dispositivos móveis como smartphones e tablets. O aparecimento de modelos com preços mais acessíveis, grande poder computacional, a mobilidade oferecida e o imediatismo das informações possibilitaram a popularização desses dispositivos em diversas camadas da sociedade, o que os transformou em uma importante ferramenta atualmente ${ }^{1,2}$.

É crescente o acesso da população à tecnologia. Segundo o Instituto Brasileiro de Geografia e Estatística (IBGE), 80,4\% das famílias utilizavam o smartphone como principal meio de acesso à internet no ano de 2016. Estes números demonstram o interesse da população brasileira pela tecnologia digital. Entende-se, diante disso, que essas tecnologias podem influenciar os hábitos dos indivíduos, como aqueles relacionados ao trabalho, ao ensino e à aprendizagem, inclusive na área da saúde, que é um importante campo de aplicação ${ }^{2-5}$.

Os smartphones trazem os aplicativos ou 'apps', que são programas desenvolvidos para serem utilizados em dispositivos desta categoria ou em tablets/iPads e/ou computadores-tablets, por exemplo ${ }^{6}$. Atualmente, há milhares de aplicativos para download na Apple Store (servidor do sistema IOS) e na Play Store (servidor do sistema Android). Realizando uma busca rápida na categoria medicina, por exemplo, é possível encontrar desde aplicativos simples como jogos até bases de dados específicas que tratam de saúde baseada em

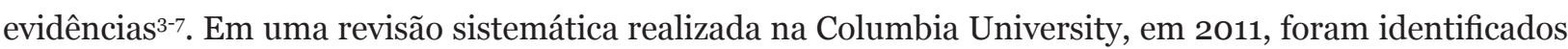
83 aplicativos móveis documentados na literatura internacional, sendo 57 direcionados aos profissionais de saúde, 11 voltados especificamente para estudantes de medicina e enfermagem e 15 para os pacientes ${ }^{8}$.

A utilização dessas ferramentas, tanto por profissionais como estudantes, tem como finalidade auxiliálos nos atendimentos diários, minimizando possíveis falhas que possam vir a ocorrer na tomada de decisão clínica por falta de acesso a informações atualizadas ${ }^{6,9,10}$. Além disso, muitos estudantes utilizam os aplicativos para aumentar a experiência perante determinado assunto acadêmico ou fazer anotações de casos, com o intuito de melhorar seu aprendizado no ambiente clínico e apresentar um bom desempenho. Inclusive, foi observado em um estudo com graduandos em medicina, que quase $90 \%$ dos participantes usavam aplicativos de celular'.

$\mathrm{O}$ uso de aplicativos quando feito de forma consciente pode trazer ótimos benefícios na conduta perante um paciente. No entanto, quando esses recursos passam a ter uma importância maior do que os pacientes em si, diminuindo assim a atenção dada aos mesmos, prejudicam a relação profissionalpaciente, sendo apontados como negativos ${ }^{1,6,7}$.

Há poucas pesquisas que verificam o uso e a influência dos aplicativos na área de saúde em estudantes de graduação, especialmente no Brasil. Devido à relevância da temática, torna-se necessário, portanto, conhecer esses aspectos para identificar quais são as contribuições positivas e/ou negativas dessas ferramentas na abordagem profissional-paciente ${ }^{1,3,6}$.

Nessa perspectiva, este estudo tem como objetivo verificar a opinião e as atitudes relatadas por estudantes concluintes de medicina a respeito do uso de aplicativos na prática clínica e no cotidiano ambulatorial de um hospital-escola universitário.

\section{Método}

Trata-se de um estudo observacional transversal analítico, oriundo de pesquisa de iniciação científica, realizado na Faculdade Pernambucana de Saúde, uma instituição de ensino superior privada localizada no município de Recife, Pernambuco. A coleta de dados foi realizada no período de setembro a dezembro de 2017, após aprovação no Comitê de Ética em Pesquisa com Seres Humanos. 
No estudo aqui apresentado foram incluídos 73 estudantes regularmente matriculados no último semestre do curso de graduação em medicina da referida instituição, que possuíam dispositivos eletrônicos com acesso a aplicativos. Os demais estudantes foram excluídos por não possuírem dispositivos de tecnologia móvel como smartphones, tablets/iPads ou qualquer outro aparelho com acesso a aplicativos.

Os estudantes, após concordarem em participar da pesquisa mediante assinatura do termo de consentimento livre e esclarecido, receberam um questionário autoaplicável. Responderam a questões objetivas sobre o tipo de dispositivo móvel que possuem, a utilização que fazem dele com intuito acadêmico, os aplicativos que mais utilizam na rotina acadêmica e sua confiabilidade. Expuseram, também, suas opiniões sobre como os aplicativos influenciam em sua rotina acadêmica por meio de bloco de assertivas organizado numa escala Likert com 5 opções de respostas para sentenças (discordo totalmente, discordo, sem opinião, concordo e concordo totalmente), sendo o número 1 correspondente à resposta 'discordo totalmente', e o número 5 correspondente a 'concordo totalmente'.

Os resultados do questionário foram expressos através de frequências absolutas e percentuais para as variáveis categóricas. Para o questionário baseado na escala Likert foram calculados os percentuais das respostas de cada sentença. Os dados foram digitados na planilha Excel e o programa utilizado para a elaboração dos cálculos estatísticos foi o SPSS (Statistical Package for the Social Sciences) versão 23. Os resultados estão apresentados em tabelas para facilitar a visualização.

\section{Resultados}

A idade dos pesquisados variou de 22 a 35 anos, mas em média tinham 26 anos. A maioria, 57,3\% (42), é do sexo feminino. Como demonstrado na Tabela 1, 95,9\% (70) dos participantes referiram possuir smartphone, 50,7\% (37) tablet e 41,1\% (30) notebook. Apenas um estudante afirmou não utilizar os dispositivos móveis com intuito acadêmico. Entre as finalidades mais comuns encontramos: 68 usam para consulta de fármacos existentes (93,2\%), 52 como fonte de estudos 71,2\%, 61 como meio para identificar a melhor terapêutica para o paciente (83,6\%), 63 para avaliação de escores clínicos (86,3\%) e 53 para ajuda em diagnósticos(72,6\%). E 59 dos participantes, equivalentes a 80,8\%, afirmaram utilizar também mídias sociais (Whatsapp, Facebook, Twitter ou outra plataforma semelhante) com intuito acadêmico. 
Tabela 1 - Distribuição dos pesquisados segundo as questões relacionadas ao uso de dispositivos móveis na rotina acadêmica

$\begin{array}{lll}\text { Variável } & \mathbf{n} & \%\end{array}$

Tipo de dispositivo que possui (1)

$\begin{array}{lll}\text { Smartphone } & 70 & 95,9\end{array}$

Tablet $\quad 37 \quad 50,7$

$\begin{array}{lrr}\text { Notebook } & 30 & 41,1\end{array}$

$\begin{array}{lll}\text { Uso de dispositivos móveis com intuito acadêmico } & 72 & 98,6\end{array}$

Finalidade do uso de dispositivos móveis com intuito acadêmico (2)
Ajuda para diagnósticos

Identificar a melhor terapêutica para o paciente $\quad 61 \quad 83,6$

Verificação/análise de protocolos $\quad 52 \quad 71,2$

Avaliação de escores clínicos $\quad 63 \quad 86,3$

Adesão farmacológica do paciente $\quad 13 \quad 17,8$

Fonte de estudos $\quad 52 \quad 71,2$

Comunicação profissional de saúde x paciente $\quad 10 \quad 13,7$

Interação profissional com profissional $\quad 32 \quad 43,8$

Consulta de fármacos existentes $\quad 68 \quad 93,2$

Verificação da condição médica do paciente $\quad 19 \quad 26,0$

Mídias (Whatsapp, Facebook, Twitter) $\quad 59 \quad 80,8$

Aplicativo mais utilizado na rotina acadêmica (2)

Multifuncionais

Artigos

Mídia social

Variados

$\begin{array}{cc}51 & 69,9 \\ 9 & 12,3 \\ 4 & 5,5 \\ 8 & 11,0\end{array}$

Como tomou conhecimento do aplicativo? ${ }^{(2)}$

Loja de aplicativos

$9 \quad 12,3$

Recomendações $\quad 52 \quad 71,2$

$\begin{array}{lrr}\text { Propaganda } & 10 & 13,7\end{array}$

$\begin{array}{lrr}\text { Internet } & 7 & 9,6\end{array}$

$\begin{array}{lrr}\text { Outro } & 6 & 8,2\end{array}$

$\begin{array}{lcc}\text { Acredita que esse aplicativo tem uma confiabilidade adequada à sua } & \\ \text { finalidade? (1) } & 72 & 98,6 \\ \text { Sim } & 0 & 0,0 \\ \text { Não } & 1 & 1,4 \\ \text { Não sabe } & 52 & 71,2\end{array}$

Fonte: Os autores (2019).

Considerando que um mesmo pesquisado poderia citar mais de uma alternativa, registra-se apenas a base para o cálculo dos percentuais e não o total

(1) Base igual a 73 pesquisados. (2) Base igual a 72 correspondente aos que utilizavam dispositivo móvel.

Ainda na Tabela 1, considerando que os participantes poderiam selecionar mais de uma opção, é possível observar que os aplicativos utilizados na rotina acadêmica por 51 deles, ou seja, pela maioria (69,9\%), eram os multifuncionais (aplicativos com diagnósticos, tratamento, clínica etc.), em seguida, os de tipos variados (aplicativos de busca, vídeos, notas, tabelas, classificação estatística internacional de doenças etc.) eram utilizados por 8 , o que corresponde a $11 \%$ e, por último, 5,5\% tanto os relacionados à anatomia do corpo humano quanto ao uso das mídias sociais (Facebook, Twitter etc.) foram relatados por 4 , equivalendo assim a 5,5\%.

Ao serem questionados sobre como tomaram conhecimento dos aplicativos que mais utilizam, como mostra na Tabela 1, 52 responderam ter sido através de recomendações, correspondendo à maior parte que 
proporcionalmente atingiu 71,2\%. Cabe destacar que 52 dos estudantes (71,2\%) costumam utilizar mais de um aplicativo na rotina acadêmica.

Sobre os resultados apresentados na Tabela 2, observa-se haver consenso, entre os estudantes entrevistados, quanto ao fato de que os profissionais de saúde devem usar aplicativos na prática clínica e que os estudantes não se prejudicam por usarem aplicativos, pois acreditam que o auxílio é positivo e que as informações obtidas são confiáveis. Em contrapartida, foi questionado, entre os participantes, se o uso de aplicativos interfere na ética e relação profissional-paciente, especialmente se o paciente tomar conhecimento do uso do aplicativo; em consequência, apenas 6,8\% dos entrevistados concordam que os profissionais sempre devem informar aos pacientes que estão sendo auxiliados por aplicativos. Também não houve consenso sobre a utilização da mídia social para comunicação com o paciente, assim como a utilização de aplicativos como fonte de atualização.

Tabela 2 - Frequências absolutas e relativas das respostas dos estudantes pesquisados pelo questionário organizado de acordo com a escala Likert

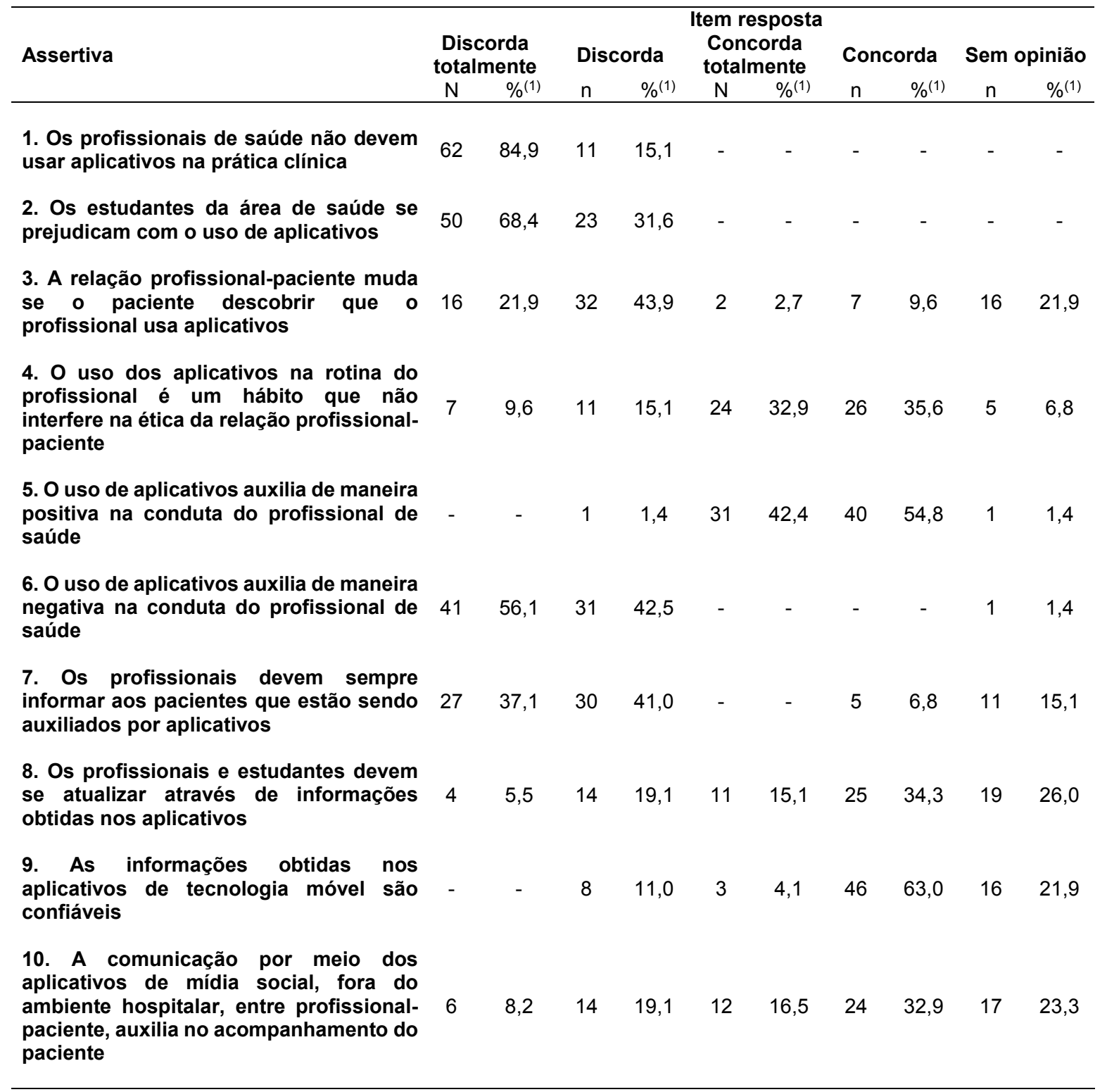

Fonte: Os autores (2018).

(1): Os valores percentuais foram obtidos com base no total (73) de pesquisados. 


\section{Discussão}

Atualmente, mais de 1,3 bilhão de aparelhos celulares estão sendo vendidos no mundo. Em 2010, os smartphones compuseram cerca de 20\% do total de aparelhos e as vendas crescem 100\% ao ano. Estimou-se o acesso a esses dispositivos por cerca de 46,7 milhões de brasileiros no final de 2015. Em 2014, esse número era de 39,7 milhões, tendo aproximadamente $67 \%$ da população brasileira fazendo uso da internet. Além de fortemente presentes na vida da população em geral, pesquisas realizadas no Reino Unido verificaram ser rotina, entre os profissionais de saúde, possuir e usar tais aparelhos como forma de melhorar a comunicação no ambiente hospitalar, ${ }^{411-14}$.

No presente estudo, a maioria dos participantes (95,9\%) referiu ser o smartphone o dispositivo móvel mais utilizado na rotina acadêmica e 98,6\% afirmaram utilizar os dispositivos móveis com intuito acadêmico. Achados expressivos também foram encontrados em outras pesquisas, como a realizada no Campus de Ciências Médicas da Universidade de Nairobi em 2015, com 88\% dos entrevistados possuindo dispositivos móveis, e 99,6\% deles utilizando com finalidade de pesquisa e aprendizado, sendo $64 \%$ relacionados à temática de saúde. Também em um estudo realizado no Canadá, em 2009, quase 90\% afirmaram utilizar smartphones e $85 \%$ deles o tinham utilizado pelo menos uma vez com intuito acadêmico ${ }^{6,12}$.

Semelhante aos nossos resultados, em relação à finalidade do uso acadêmico de dispositivos eletrônicos, mais de $50 \%$ dos estudantes a partir do $3^{\circ}$ ano do curso de medicina e residentes, num estudo realizado em Seattle, Estados Unidos, em 2013, referiram as procuras por fármacos, protocolos, uso de calculadoras clínicas e ajuda para diagnósticos, como as mais comuns ${ }^{15}$. Já em estudo realizado no Hospital Educacional Azzahra no Isfahan (Irã), no ano de 2013, sobre a consciência do uso dos aplicativos na rotina de estudantes de medicina, 38,4\% dos participantes referiram usar aplicativos para auxiliar em diagnósticos, 40,1\% para auxiliar no tratamento e $31,8 \%$ relacionados a fonte de aprendizados ${ }^{16}$.

Da mesma forma, na Universidade de Nairobi, em 2015, quase a totalidade dos estudantes (99\%) utilizam mídias sociais com intuito acadêmico, como Whatsapp e Facebook, uso este também relatado por $80,8 \%(n=59)$ dos participantes da nossa pesquisa. No mesmo trabalho foram relatados como aplicativos mais acessados os relativos ao gerenciamento de doenças (88\%), referido aqui no grupo dos multifuncionais; dicionários médicos (87\%), referência laboratorial (81\%) e consulta de fármacos existentes (73\%) enquadrados como aplicativos de tipos variados. Também foi verificado por pesquisadores de uma universidade em São Paulo, o uso de redes sociais com intuito de aprendizado por $61 \%$ dos estudantes participantes ${ }^{17}$.

Os aplicativos mais utilizados na rotina acadêmica dos nossos estudantes foram da categoria multifuncional (Medscape ${ }^{\mathrm{TM}}$, Whitebook $\AA$, entre outros), com 69,9\% (51). Em conformidade com esses resultados, estudantes de medicina da Universidade de Nairobi e da Universidade de Birmingham no Reino Unido, em 2012, listaram como aplicativo mais utilizado o Medscape $^{\mathrm{TM}}$ (66\%). Pesquisa semelhante desenvolvida na Universidade Estadual de Farmácia de Ohio ainda nos faz refletir a respeito da associação entre a preferência de escolha do aplicativo e a área de atuação profissional, ao revelar que os aplicativos para consulta de drogas são mais acessados pelos estudantes/profissionais da área de farmácia ${ }^{12,14,18}$.

Ao serem indagados sobre como tomaram conhecimento dos referidos aplicativos, o principal modo de descoberta (71,2\%) foi através de terceiros (colegas, mídias sociais etc.), assim como relatado no mencionado estudo desenvolvido em Ohio em 2016. Segundo um guia sobre marketing e loja de aplicativos, 70\% das pessoas afirmam ler pelo menos um comentário acerca do programa antes de baixá-lo, ou seja, quanto melhor classificado e mais recomendado, mais downloads são realizados ${ }^{14,19}$.

Os resultados referentes à opinião dos participantes deste estudo corroboram com algumas informações de uma pesquisa realizada pela Universidade Estadual do Rio de Janeiro, sendo demonstrada satisfação dos alunos com a proposta de utilização de aplicativos móveis como auxílio no ensino e na aprendizagem em 
saúde ${ }^{20}$. Inclusive, o uso de dispositivos eletrônicos como celulares, aparelhos de monitoração de pacientes, entre outros dispositivos com acesso à internet sem fio, na prática médica e pela população, relacionados à saúde foi reconhecido recentemente, em 2012, pela Organização Mundial de Saúde (OMS) e definido como "Mobile Health (mHealth)", conceito este ainda sem regulamentação no Brasil 5,8 .

Apesar da aceitação mundial do uso de aplicativos de tecnologia móvel na rotina clínica ser vista de maneira positiva, nossos entrevistados ainda questionam até que ponto os aplicativos podem fornecer benefícios para os profissionais de saúde. No estudo já citado da Universidade de Alberta, foi relatado por $37 \%$ dos entrevistados que o uso da tecnologia tem o potencial de comprometer o comportamento profissional, já que os usos acadêmico e pessoal podem se contrapor ${ }^{6}$. Na nossa pesquisa, muitos dos estudantes questionados sobre essa questão acreditam que o uso dos aplicativos na rotina interfere na relação profissional de saúde-paciente.

Quanto à confiabilidade dos aplicativos utilizados na rotina acadêmica, apesar de quase $100 \%$ confiar, na análise das opiniões pela escala Likert verificamos que existe preocupação com o seu uso. Achados semelhantes foram trazidos por estudos realizados no Canadá com alunos de medicina e nos Estados Unidos com alunos do curso de farmácia ${ }^{6,14}$.

Como alternativa, na Universidade de Nairobi, por exemplo, foi verificado que cerca de $50 \%$ dos estudantes de medicina relataram que primeiro instalam os aplicativos para depois analisarem sua confiabilidade ${ }^{12}$. No entanto, atitudes como esta não impedem o uso, já que muitos consideram os smartphones como facilitadores do aprendizado, por proverem acesso rápido ao conteúdo e por suplementar outros meios de pesquisa, otimizando o tempo. Até porque, observa-se que muitos estudantes não têm ainda maturidade intelectual e conhecimento teórico-prático suficientes para reconhecer a confiabilidade de um aplicativo. Estudantes de farmácia, por exemplo, na pesquisa já anteriormente citada em Ohio, reconheceram suas inabilidades para identificar quando é apropriado o uso do celular na prática clínica ${ }^{6,14}$.

Por fim, é importante ressaltar que, por ser um estudo de opinião de corte transversal, as informações desta pesquisa são relatadas, não tendo sido confirmado se, de fato, os estudantes utilizam os aplicativos mencionados no questionário na sua rotina clínica.

\section{Conclusão}

Conclui-se que o uso de dispositivos eletrônicos com acesso à internet na graduação em medicina é uma realidade, sendo o smartphone o principal meio. Apresenta potencialidades e fragilidades, podendo ser utilizado de maneira coerente e cautelosa a favor da prática profissional, como também comprometendo a relação profissional de saúde-paciente, quando não há confiabilidade suficiente dos dados obtidos para aprendizagem. É necessário, no entanto, mais estudos na área para aprofundar conhecimentos a respeito do uso dos aplicativos de tecnologia móvel na rotina clínica, especialmente entre as diferentes áreas da saúde, não só com estudantes, mas também com profissionais como público-alvo.

\section{Referências}

1. Salles AA. Transformações na relação médico-paciente na era da informatização. 2010;18(1):49-60.

2. Silva GHP, Silva JVL, Ruppert GCS, I RDP. Desenvolvimento de aplicativos para visualização de imagens médicas em dispositivos móveis. XXXII Congresso da Sociedade Brasileira de Computação; Curitiba: UFPR; 2012.

3. Oliveira ARF, Alencar MSM. O uso de aplicativos de saúde para dispositivos móveis como fontes de informação e educação em saúde. RDBCI: R. Digit. Bibliotecon. Ci. Inf [Internet]. 2017 [citado em 2019 jul. 22];15(1):234. doi: https://doi.org/10.20396/rdbci.v15i1.8648137. 
4. Caivano S, Ferreira BJ, Domene SMA. Avaliação da usabilidade do Guia Alimentar Digital móvel segundo a percepção dos usuários. Ci. Saude Coletiva [Internet]. 2014 [citado em 2019 jul. 22]; 19(5):1437-46. doi: http://dx.doi.org/10.1590/1413-81232014195.13932013.

5. Cruz DIP, Dias RRD, Martins WS, Gandolfi VF, Elizandro P. O uso das mídias digitais na educação em saúde. Cad FUCAMP [Internet]. 2011[citado em 2019 jul. 22]; 10(13):130-42. Disponível em: http://www. fucamp.edu.br/editora/index.php/cadernos/article/view/215.

6. Wallace S, Clark M, White J. 'It's on my iPhone': attitudes to the use of mobile computing devices in medical education, a mixed-methods study. BMJ Open [Internet]. 2012 [citado em 2019 jul. 22];2(4):e001099-e001099. doi: http://dx.doi.org/10.1136/bmjopen-2012-001099.

7. Tibes CMDS, Dias JD, Zem-Mascarenhas SH. Mobile applications developed for the health sector in Brazil: an integrative literature review. REME R. Min. Enferm. [Internet]. 2014 [citado em 2019 jul. 22];18(2):471-78. doi: http://dx.doi.org/10.5935/1415-2762.20140035.

8. Mosa ASM, Yoo I, Sheets L. A systematic review of healthcare applications for smartphones. BMC Med. Inform. Decis. Mak. [Internet]. 2012 [citado em 2019 jul. 22];12(1):67. doi: http://dx.doi.org/10.1186/14726947-12-67.

9. Lázaro HA, Eduardo C, Leão G. Uso de aplicativos móveis para auxílio no atendimento das vítimas de queimaduras Use of mobile phone app to help in treatment of burn victims. R. Bras. Queimaduras. 2013;12(4): 286-88.

10. Sarno F, Canella DS, Bandoni DH. Mobile health e excesso de peso: uma revisão sistemática. $R$. panam salud pública [Internet]. 2014[citado em 2019 jul. 22]; 35: 424-31. Disponível em: http://www. scielosp.org/pdf/rpsp/v35n5-6/18.pdf.

11. Raiman L, Antbring R, Mahmood A. WhatsApp messenger as a tool to supplement medical education for medical students on clinical attachment. BMC Med Educ [Internet]. 2017 [citado em 2019 jul. 22];17(1):7. doi: http://dx.doi.org/10.1186/s12909-017-0855-x.

12. Masika MM, Omondi GB, Natembeya DS, Mugane EM, Bosire KO, Kibwage IO. Use of mobile learning technology among final year medical students in Kenya. Pan Afr Med J [Internet]. 2015 [citado em 2019 jul. 22]; 21: 1-12. doi: http://dx.doi.org/10.11604/pamj.2015.21.127.6185.

13. Formagini TDB, Ervilha RR, Machado NM, Andrade BABB de, Gomide HP, Ronzani TM. A review of smartphone apps for smoking cessation available in Portuguese. TT - Revisão dos aplicativos de smartphones para cessação do tabagismo disponíveis em língua portuguesa. Cad Saude Publica [Internet]. 2017 [citado em 2019 jul. 22];33(2):1. doi: http://dx.doi.org/10.1590/0102-311X00178215.

14. Rodis J, Aungst TD, Brown N V, Cui Y, Tam L. Enhancing Pharmacy Student Learning and Perceptions of Medical Apps. JMIR mHealth uHealth [Internet]. 2016 [citado em 2019 jul. 22]; 4(2):e55. doi: http:// dx.doi.org/10.2196/mhealth.4843.

15. Boruff JT, Storie D. Mobile devices in medicine: a survey of how medical students, residents, and faculty use smartphones and other mobile devices to find information. J Med Libr Assoc [Internet]. 2014 [citado em 2019 jul. 22]; 102(1):22-30. doi: http://dx.doi.org/10.3163/1536-5050.102.1.006.

16. Ehteshami A, Hachesu PR, Esfahani MK, Rezazadeh E. Awareness and using of medical students about mobile health technology in clinical areas. Acta Inform Medica [Internet]. 2013 [citado em 2019 jul. 22];21(2):109-112. doi: http://dx.doi.org/http://dx.doi.org/10.5455/aim.2013.21.109-12.

17. Matthiesen RC. Análise do uso de redes sociais para ensino-aprendizagem de alunos de curso superior. 2010;13(16):73-92.

18. Robinson $\mathrm{T}$, Cronin $\mathrm{T}$, Ibrahim $\mathrm{H}$, et al. Smartphone use and acceptability among clinical medical students: A questionnaire-based study. J Med Syst [Internet]. 2013 [citado em 2019 jul. 22];37(3):1-7. doi: http://dx.doi.org/10.1007/s10916-013-9936-5.

19. Walz A, Ganguly R. The Mobile Marketer's Guide To App Store Ratings \& Reviews. 2015 Editi. Seattle: Apptentive; 2015.

20. Mercês JMR, Redeiro MMP. A Importância dos dispositivos móveis como estratégia para a formação o desenvolvimento de profissionais de saúde. 22 Ciaed - Abed. 2016. http://www.abed.org.br/ congresso2016/trabalhos/306.pdf. 